\title{
Orbital Myositis with Sudden Visual Loss: A Case Study in Niamey, Niger
}

\author{
Abba Kaka H.Y., \\ Ophthalmology Department, Niamey National Hospital, Niger \\ Guirou N., \\ African Institute of Tropical Ophthalmology Bamako, Mali \\ Moussa M., \\ Stomatology Department, Niamey National Hospital, Niger
}

\section{Boubacar M., Amza A.,}

Ophthalmology Department, Lamorde National Hospital Niamey, Niger

\section{Doi: 10.19044/esj.2018.v14n36p45 URL:http://dx.doi.org/10.19044/esj.2018.v14n36p45}

\begin{abstract}
This paper focuses on presenting a case study of three cases of orbital myositis in one male and two female adult patients. All patients presented sudden loss of vision and proptosis at the initial stage. Ophthalmological examination outlined optic nerve atrophie. The diagnosis of all cases was confirmed with a CT scan which showed specific muscle inflammation. The treatment was successfully carried out using bolus doses of corticosteroids relayed with oral prednisolone for over 6 weeks. Six (6) months follow-up, however, did not show any relapse in all the cases.
\end{abstract}

Keywords: Orbital myositis, extra-ocular muscles, Niger

\section{Introduction}

Orbital myositis $(\mathrm{OM})$ is an inflammatory process affecting one or more oculomotor muscle and first merged with the term "Orbital pseudotumor" in 1903 (Gleason, 1903).

Orbital Myositis is mainly caused by non-thyroid inflammatory diseases; or it could be idiopathic. Vision is generally spared but in some rare cases, lowering of vision could be the alarming sign (Dubey et al., 2009). To the author's knowledge, psychiatric symptom is not known to result from Orbital myositis. Thus, this study presents reports of patients who were referred to an ophthalmology emergency clinic in Niamey, Niger, for proptosis associated to sudden visual loss. 


\section{Patients and Methods}

The study was carried out on 3 adult patients two females and one male- who were referred to the ophthalmology emergency clinic for proptosis and sudden loss of vision.

\section{Case No 1.}

Mr. M.A is a farmer of 55 years. He complained of low vision, painful eye movement and proptosis; all on the right eye. These symptoms were accompanied by a low grade fever. There was no past history of ocular or thyroid disease. On physical evaluation, he had a preserved general status, a fever of $39.9^{\circ} \mathrm{C}$, blood pressure was $130 / 80$, the pulse rate was 90 and a respiratory rate of 30 . On external eye examination, there was a non axial (down and out) painful and reducible right eye grade 2 proptosis, and conjunctival chemosis. There was no light perception on the affected eye and the pupil was dilated with no response to light projection. When funduscopy was conducted, atrophic disc pallor was observed. The left eye was unaffected. Biological findings were hyperleukocytosis (14 $000 \mathrm{WBC})$, elevated CRP level $(76 \mathrm{mg} / \mathrm{dl})$, normal thyroid hormones levels: T3: 2.6 (2.50- 3.90umol/L); T4: 1.06 (0.85-1.76 ng/dL); TSH: 2.03 (0.27-4.68uIU/mL); anti-nuclear antibody level was normal (normal level 1:80); normal blood sugar $(1.02 \mathrm{mmol} / \mathrm{l})$, Rheumatoid factors at $08(\leq 14 \mathrm{IU} / \mathrm{mL})$.

The CT scan showed an important hypertrophy of superior rectus muscle with a normal optic nerve sheath (Figure 1). Its treatment was an association of intravenous antibiotics (cephalosporin of third generation) and corticosteroids (methyl-prednisolone a bolus stating dose and a daily dose of $1 \mathrm{mg} / \mathrm{kg} /$ day). Remission of proptosis was followed by a normalization of behaviour. A follow-up of about 6 months showed no actual relapse.


Figure 1a and b. Enlarged superior rectus muscle belly (blue arrow) 


\section{Case No 2.}

Mrs. H.A is a 55 years old house wife who was transferred to the ophthalmology emergency clinic from the psychiatric ward for painful red left eye since about 10 days. She started having delirium and became violent 5 days after the inception of pain and loss of vision on the left eye. She had no past history of psychiatric, ocular or thyroid disease. An ophthtalmoscopic examination revealed that the left eye visual acuity was of no light perception. External examination found a grade III proptosis associated to a complete ophthalmoplegia. Slit lamp examination out lined a conjunctival chemosis, dilated non reactive pupil, and an important disc pallor at funduscopy. The right eye had no abnormality. Biological check-up showed only an elevated CRP 100mg/dl, ESR 60 at first hour, while the thyroid hormones levels were normal (T3:3; T4 :1.5; TSH: 3). CT scan of the orbit showed thickening of all the extra-ocular muscles (Figure 2). No auto-antibody was found. Its treatment was with a bolus dose of prednisolone, followed with a daily dose at $1 \mathrm{mg} / \mathrm{kg} /$ day. Treatment lasted six weeks and was followed up for 6 months with no relapse. Delirium was over after a week of treatment; proptosis was completely over but there was no visual recovery.

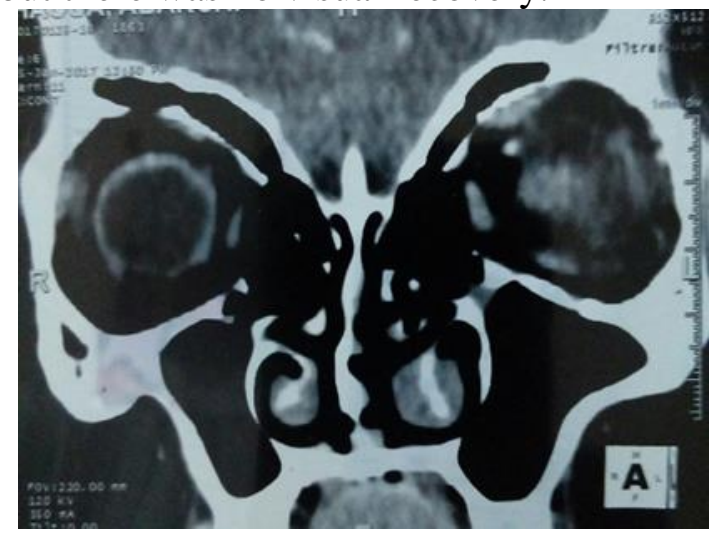

Figure 2. Enlargement of extra-ocular muscles on left eye

\section{Case No 3.}

Mrs. A.A is a young lady of 22 years. She was referred from the endocrinology department for proptosis, lowering of sight and instable type 1 diabetes. On examination, she had a visual acuity of light perception at the right eye and 10/10 at the left eye. External eye examination evidenced a limitation and painful movements of the rectus medial on the right side; while fundus examination showed a pale atrophic optic disc. Meanwhile, the left eye had no notable abnormality. CT scan evidenced an inflamed and enlarged rectus medial muscle, ESR level was elevated 65mg/dl, CRP 90 at first hour, and there was no auto antibodies found nor was there an elevated thyroid hormones (T3:2.9umol/L; T4:0.96ng/dL; TSH:2.8IU/mL). The diabetes was 
difficult to stabilise and was, thus, placed on insulin pump. The treatment consisted of bolus doses of methyl-prednisolone for 3 days followed with oral hydrocortisone for over 4 weeks and, afterwards, gradual degressive doses for over 6 weeks. Remission was completed within 6 weeks of treatment with no remission till date.


Figure 3 a and b. Enlarged medial rectus muscle

\section{Discussion}

Idiopathic orbital myositis (OM) is classified as a subtype of nonspecific orbital inflammation that usually involves the extra-ocular muscles (EOMs). It is seen mostly in young to middle-aged adults around 40 years. (with range 7 to 80 years). There is an admitted ratio of 2 to 1 patients of orbital Myositis with female predominance. It is unanimously argued in the literature that females are more affected, as seen in this study (Hankey, 1937; Trokel et al., 1979; Dylewski, 2001; Byrne et al., 1992; Purcell et al., 1981; Espinoza et al., 2010; Smith et al., 1992; Sobrin et al., 2007). The rectus media is mostly affected. This is closely followed by the superior rectus and rarely oblique muscle (Trokel et al., 1979; Dylewski et al., 2001). The affection is mostly unilateral and many muscles could be affected at a time. Radiological images of affected muscles revealed the enlargement of muscle bellies along with thickened tendons (Byrne et al., 1992; Trokel et al., 1979). The etiology and pathogenesis of this entity is currently unknown. Both infectious and immune-mediated etiologies were implicated. Following streptococcal pharyngitis, orbital myositis as well as many other systemic diseases such as Crohn's disease, rheumatoid arthritis, myasthenia gravis, and ankylosing spondylitis were reported by Purcell and Taulbee (Purcell et al., 1981; Espinoza et al., 2010; Woo et al., 2001). Mombaerts et al. (1997) found in their study that $10 \%$ of their patients with $\mathrm{OM}$ also had a concurrent autoimmune disease. Auto antibodies against oculomotor muscles had been reported by authors such as Atabay et al. (1995). However, these antibodies were also seen 
in thyroid orbitopathy. Additionally, the typical unilateral presentation of OM argues against this type of autoimmunity as being the primary mechanism of OM (Atabay et al., 1995). Symptoms are mostly pain in adult and this occurs in 58-69\% of patients, followed by diplopia at 31-38\%; periorbital edema chemosis and proptosis have also been reported (Berger et al., 1996; Ding et al., 2011). Therefore, external examination of affected patients should emphasize on lid assessment for retraction/lid lag/lagophthalmos, orbital assessment (proptosis), extra-ocular muscles (restriction), globe (injection/chemosis), and optic nerve function (visual acuity/color plates/relative afferent pupillary defect). Vision is usually spared, progressive lowering, and, sometimes, total loss could be found (Gleason et al., 1903; Dubey et al., 2009). To the author's knowledge, psychiatric manifestations following symptoms of idiopathic orbital myositis have not been reported in the literature. A satisfactory therapeutic consensus is lacking for this pathology. Other methods of treatment such as corticosteroid therapy, immunosuppressive drugs, radiotherapy and sometimes surgery were reported in the literature (Recai et al., 2011). Corticosteroid drugs at the dose of 1.0 to $1.5 \mathrm{mg} / \mathrm{kg} / \mathrm{d}$ are the first line of treatment with quite a good response rate in most patients. This is the protocol we used in our cases with complete remission. Antimetabolites, such as azathioprine, methotrexate, mycophenolate mofetil are frequently used (Lacey et al., 1999; Franzco et al., 2006; Harris et al., 2006). Recently, the use of tumor necrosis factor, alpha blocker and new monoclonal antibodies were reported (Recai et al., 2011; Harris et al., 2006). In some severe cases, high dose of intravenous immunoglobulin or rituximab infusion may be helpful (Franzco et al., 2006; Recai et al., 2011; Lacey et al., 1999; Dalakas et al., 2006).

\section{Conclusion}

Orbital myositis is a rare affection of the extra-ocular muscles which is mostly idiopathic. Visual loss is rare and dramatic because it is irreversible. Corticosteroids are the first line of treatment even though there is no standardized line of treatment. Prompt corticotherapy and prolonged follow up may ameliorate the prognosis of this disease.

\section{References:}

1. Atabay, C., Tyutyunikov, A., Scalise, D. et al. (1995). Serum antibodies reactive with eye muscle membrane antigens are detected in patients with nonspecific orbital inflammation. Ophthalmology; 102:145-153.

2. Berger, JW., Rubin, PAD., \& Jakobiec, FA. (1996). Pediatric orbital pseudotumor: case report and review of the literature. Int Ophthalmol Clin 1996;36:161-177. 
3. Byrne, SP. \& Green, RL. (1992). Ultrasound of the Eye and Orbit. St Louis: CV Mosby:353-92.

4. Dalakas, MC. (2006). Therapeutic targets in patients with inflammatory myopathies: present approaches and a look to the future. Neuromuscl Disord. 16:223-36.

5. Ding, ZX., Lip, G., \& Chong, V. (2011). Idiopathic orbital pseudotumor. Clinical Radiology; 66:886-892.

6. Dubey, A., Eidsness, R., \& Koul, R. (2009). Idiopathic orbital myositis and review of literature. The Internet Journal of Ophthalmology and Visual Science. 8, 2.

7. Dylewski, JS., Drummond, R., \& Townsend, T. (2001). Orbital myositis complicating sinusitis: Case report and review. Can J Infect Dis; 12(1):51-53.

8. Espinoza, GM. (2010). Orbital Inflammatory Pseudotumors: Etiology, Differential, Diagnosis, and Management. Curr Rheumatol Rep 2010;12:443-447.

9. Franzco, LL., Suhler, EB., \& Smith, JR. (2006). Biologic therapies for inflammatory eye disease. Clin Experiment Ophthalmol; 34:365-74.

10. Gleason, JE. (1903). Idiopathic myositis involving the extraocular muscles. Ophthalmic Rec;12: 471-8.

11. Hankey, GJ., Silbert, PL., Edis, RH., \& Nicoll, AM. (1987). Orbital myositis: a study of six cases. Aust N Z J Med; 17:585-91.

12. Harris, GJ. (2006). Idiopathic orbital inflammation: a pathogenetic construct and treatment strategy. Ophthal Plast Reconstr Surg. 2006;22:79-86.

13. Lacey, B., Chang, W., \& Rootman, J. (1999). Non thyroid causes of extraocular muscle disease. Surv Ophthalmol; 44:187-213.

14. Mombaerts, I. \& Koornneef, L. (1997). Current status in the treatment of orbital myositis. Ophthalmology 1997;104(3):402-408.

15. Purcell, JJ. \& Taulbee, WA. (1981). Orbital myositis after upper respiratory tract infection. Arch Ophthalmol 1981;99:437-438.

16. Recai Turkoglu, Naci Balak. Atypical Presentation of Orbital Pseudotumor with Visual Loss as an Initial Manifestation. J Clin Neurol 2011;7:50-52.

17. Serop, S., Vianna, RN., Claeys, M., \& DE Laey, JJ. (1994). Orbital myositis secondary to systemic lupus erythematosus. Acta Ophthalmol; 72:520-523.

18. Smith, JW. (1992). Orbital pseudotumor and Crohn's disease. Am J Gastroenterol; 87(3):405-406.

19. Sobrin, L., Kim, E., Christen, W. et al. (2007). Infliximab therapy for the treatment of refractory ocular inflammatory disease. Arch Ophthalmol. 2007;125:895-900. 
20. Trokel, SL. \& Hilal, SK. (1979). Recognition and differential diagnosis of enlarged extra ocular muscles in computed tomography. Am J Ophthalmol; 87:503-512.

21. Van de Mosselaer, G., Deuren, HV., Dewolf-Peeters, C. et al. (1980). Pseudotumor orbitae and myasthenia gravis. Arch Ophthalmol; 98:1621-1622.

22. Woo, TL., Francis, IC., Wilcsek, GA., Coroneo, MT., McNab, AA., \& Sullivan, TJ. (2001). Australian orbital and adnexal Wegner's granulomatosis. Ophthalmology;108: 1535-1543. 